\title{
Semiarcs with a long secant in $\mathrm{PG}(2, q)$
}

\author{
Bence Csajbók ${ }^{*} \quad$ Tamás Héger $^{\dagger} \quad$ György Kiss ${ }^{\ddagger}$
}

full screen

close

Abstract

A $t$-semiarc is a point set $\mathcal{S}_{t}$ with the property that the number of tangent lines to $\mathcal{S}_{t}$ at each of its points is $t$. We show that if a small $t$-semiarc $\mathcal{S}_{t}$ in $\operatorname{PG}(2, q)$ has a large collinear subset $\mathcal{K}$, then the tangents to $\mathcal{S}_{t}$ at the points of $\mathcal{K}$ can be blocked by $t$ points not in $\mathcal{K}$. In fact, we give a more general result for small point sets with less uniform tangent distribution. We show that in $\operatorname{PG}(2, q)$ small $t$-semiarcs are related to certain small blocking sets and give some characterization theorems for small semiarcs with large collinear subsets.

Keywords : finite plane, semiarc, semioval, blocking set, Szőnyi-Weiner Lemma MSC 2010: 51E20, 51E21

\section{Introduction}

Ovals, $k$-arcs and semiovals of finite projective planes are interesting geometric structures which also have applications to coding theory and cryptography. For details we refer the reader to [3, 14, 22, 24, 27].

Semiarcs are natural generalizations of arcs. Throughout the paper $\Pi_{q}$ denotes an arbitrary projective plane of order $q$. By $\operatorname{PG}(2, q)$ and $\operatorname{AG}(2, q)$ we denote the desarguesian projective and affine planes. A non-empty point set

\footnotetext{
*Author was supported by the Hungarian National Foundation for Scientific Research (OTKA), Grant No. K 81310.

${ }^{\dagger}$ Author was supported by the Hungarian National Foundation for Scientific Research (OTKA), Grant No. K 81310 and by ERC Grant No. 227701 DISCRETECONT.

${ }^{\ddagger}$ Author was supported by the Hungarian National Foundation for Scientific Research (OTKA), Grant No. K 81310 and by the Slovenian-Hungarian Intergovernmental Scientific and Technological Cooperation Project, Grant No. TÉT 10-1-2011-0606.
} 






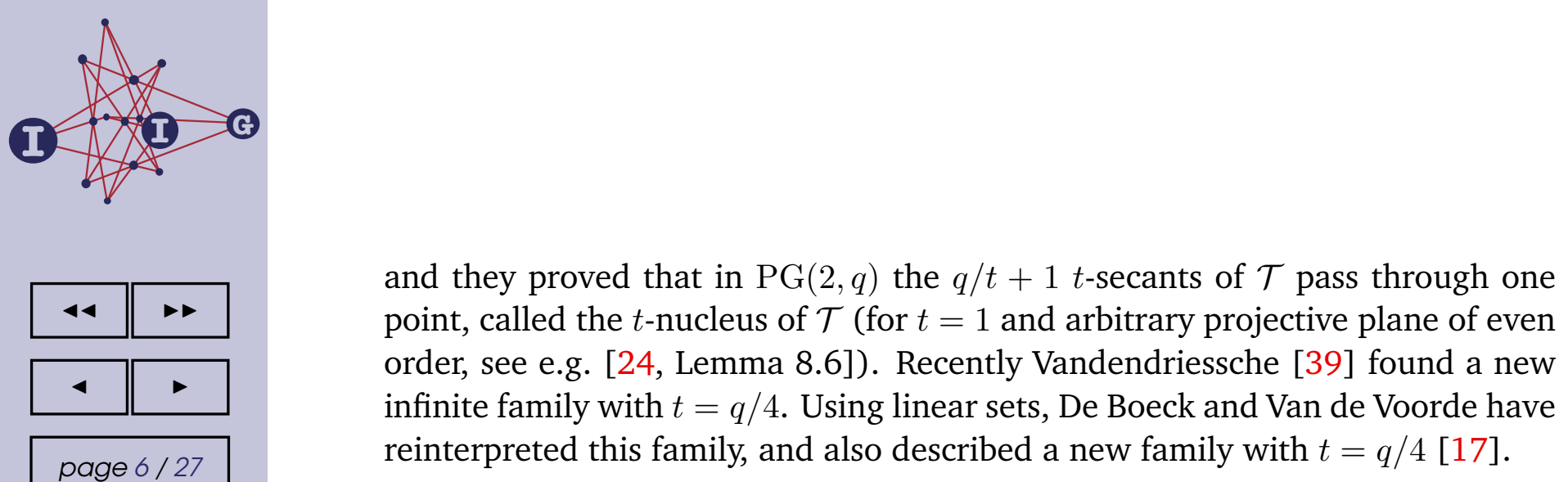

and they proved that in $\operatorname{PG}(2, q)$ the $q / t+1 t$-secants of $\mathcal{T}$ pass through one point, called the $t$-nucleus of $\mathcal{T}$ (for $t=1$ and arbitrary projective plane of even order, see e.g. [24, Lemma 8.6]). Recently Vandendriessche [39] found a new reinterpreted this family, and also described a new family with $t=q / 4$ [17].

Example 2.8. Let $\mathcal{T}$ be a $(q+\tau, \tau)$-arc of type $(0,2, \tau)$ in $\Pi_{q}$. Delete $1 \leq r<$ $\tau-2$ points from each of the $\tau$-secants of $\mathcal{T}$. The remaining points form a tight $t$-semiarc with $q / \tau+1$ maximal secants, $t=r q / \tau$ and $\kappa()=\tau-r$.

Since $(q+q / 2, q / 2)$-arcs of type $(0,2, q / 2)$ exist in $\mathrm{PG}(2, q), q$ even, this construction gives $t$-semiarcs for each $t \leq q-6, t$ even.

The so-called direction problem is closely related to tight semiarcs. We briefly collect the basic definitions and some results about this problem. Consider $\mathrm{PG}(2, q)=\operatorname{AG}(2, q) \cup \ell_{\infty}$. Let $\mathcal{U}$ be a set of points of $\mathrm{AG}(2, q)$. A point $P$ of $\ell_{\infty}$ is called a direction determined by $\mathcal{U}$ if there is a line through $P$ that contains at least two points of $\mathcal{U}$. The set of directions determined by $\mathcal{U}$ is denoted by $D_{\mathcal{U}}$. If $|\mathcal{U}|=q$, then $\mathcal{U} \cup D_{\mathcal{U}}$ is a blocking set of Rédei type. If $Y_{\infty} \notin D_{\mathcal{U}}$, then $\mathcal{U}$ can be considered as a graph of a function. Note that all these definitions make sense in non-desarguesian planes as well. Using these notions, we first give a general example of tight semiarcs.

Example 2.9. Let $\ell$ be a line of $\Pi_{q}$, and let $\mathcal{U}$ be a set of $m<q$ points in the affine plane $\Pi_{q} \backslash \ell$. Consider directions with respect to $\ell=\ell_{\infty}$. Assume $\left|D_{\mathcal{U}}\right|<m$, and denote $q-m$ by $t$. Let $\bar{D}=\ell_{\infty} \backslash D_{\mathcal{U}}$ and let $\mathcal{T} \subset \bar{D}$ be a set of $t$ points. Suppose that $\mathcal{U} \cup D_{\mathcal{U}}$ does not have 2-secants. Then the set $\mathcal{S}_{t}=\mathcal{U} \cup(\bar{D} \backslash \mathcal{T})$ is a tight $t$-semiarc with $\kappa\left(\mathcal{S}_{t}\right)=m-\left|D_{\mathcal{U}}\right|+1$.

Proof. As $\left|\ell_{\infty} \cap \mathcal{S}_{t}\right|=q+1-\left|D_{\mathcal{U}}\right|-t=m-\left|D_{\mathcal{U}}\right|+1>1, \ell_{\infty}$ is not tangent to $\mathcal{S}_{t}$. If $P \in \bar{D} \backslash \mathcal{T}$, then all lines through $P$, except $\ell_{\infty}$, intersect $\mathcal{U}$ in either zero or one point, hence the number of tangents through $P$ to $\mathcal{S}_{t}$ is $q-|\mathcal{U}|=t$. Now let $P \in \mathcal{U}$, and consider a line $\ell$ through $P$. According to whether $\ell$ intersects $\ell_{\infty}$ in $D_{\mathcal{U}}, \bar{D} \backslash \mathcal{T}$ or $\mathcal{T},\left|\ell \cap \mathcal{S}_{t}\right|$ is at least two, exactly two or exactly one, respectively. Thus there pass precisely $|\mathcal{T}|=t$ tangents to $\mathcal{S}_{t}$ through $P$.

We will consider two particular examples.

Example 2.10 (Altered Baer subplane). Let $\Pi_{\sqrt{q}}$ be a Baer subplane in the projective plane $\Pi_{q}, q \geq 9$, and let $\ell$ be an extended line of $\Pi_{\sqrt{q}}$. Let $\mathcal{P}$ be a set of $1 \leq t \leq q-\sqrt{q}-2$ points in $\Pi_{\sqrt{q}} \backslash \ell$ such that no line intersects $\mathcal{P}$ in exactly $\sqrt{q}-1$ points. For example, a $(t, \sqrt{q}-2)$-arc is a good choice for $\mathcal{P}$. Example 2.9 with $\mathcal{U}=\Pi_{\sqrt{q}} \backslash(\ell \cup \mathcal{P})$ gives a tight $t$-semiarc with $\kappa()=q-\sqrt{q}-t$. 
We also need the following lemma.

Lemma 2.15. Let $z$ and $t$ be two positive integers such that $z \geq 3$ and $t \leq$ $\sqrt{q(z-1) / z}$. Let $\mathcal{U} \subset \mathrm{AG}(2, q)$ be a set of $q-t$ affine points, and let $E \subseteq F$ be two sets of directions satisfying the following properties:

1. there are at least $t$ tangents to $\mathcal{U}$ with direction in $F$ through each point of $\mathcal{U}$;

go back

full screen

close

2. there exists a suitable set of $t$ affine points, $\mathcal{P}$, such that $\mathcal{U} \cap \mathcal{P}=\emptyset$ and each tangent to $\mathcal{U}$ with direction not in $E$ intersects $\mathcal{U} \cup \mathcal{P}$ in $0(\bmod z)$ points.

Then $|E| \geq t$.

Proof. If $\ell$ is a tangent to $\mathcal{U}$ intersecting $F \backslash E$, then $|\mathcal{P} \cap \ell| \equiv-1(\bmod z)$. The maximum number of such tangent lines is $\frac{t(t-1)}{(z-1)(z-2)}$. Hence at least $(q-t) t-$ $\frac{t(t-1)}{(z-1)(z-2)}$ tangents to $\mathcal{U}$ have direction in $E$. This implies

$$
|E| q \geq(q-t) t-\frac{t(t-1)}{(z-1)(z-2)}, \quad \text { thus } \quad(|E|-t) q \geq-t^{2}-\frac{t(t-1)}{(z-1)(z-2)}
$$

If $|E|-t$ is a negative integer, then this inequality gives $q<t^{2} \frac{(z-1)(z-2)+1}{(z-1)(z-2)} \leq$ $t^{2} z /(z-1)$, contradicting the assumption $t \leq \sqrt{q(z-1) / z}$.

Theorem 2.16. Let be a tight $t$-semiarc in $\mathrm{PG}(2, q), q=p^{h}$, $p$ prime. Suppose that one of the following conditions hold:

- $t=1, q>4$ and $\kappa\left(\mathcal{S}_{1}\right)>(q-1) / 2$, or

- $2 \leq t \leq \alpha \sqrt{q}$ and $\kappa()>\alpha(q+1)$ for some $1 / 2 \leq \alpha \leq \sqrt{(p-1) / p}$ if $p$ is an odd prime, and $1 / 2 \leq \alpha \leq \sqrt{3} / 2$ if $p=2$.

Then is an altered Rédei type blocking set.

Proof. Let $k=\kappa()$ and let $\ell$ be a $k$-secant of . Take $\ell$ as the line at infinity and let $\mathcal{U}=\backslash \ell \subseteq \operatorname{AG}(2, q)$. The directions in $\cap \ell$ are not determined by $\mathcal{U}$, hence $\left|D_{\mathcal{U}}\right| \leq q+1-k$. We can apply Theorem 2.13 when $t=1$; if $t \geq 2$, then the conditions of Theorem 2.14 hold since $|\mathcal{U}|=q-t, t \leq \alpha \sqrt{q}$ and $\left|D_{\mathcal{U}}\right|<(q+1)(1-\alpha)$. Let $\mathcal{P}=\left\{P_{1}, P_{2}, \ldots, P_{t}\right\}$ be a set of $t$ points such that $\mathcal{U} \cup \mathcal{P}$ determines the same directions as $\mathcal{U}$.

First consider the case $t \geq 2$. We have $\left|D_{\mathcal{U}}\right|<(q+1) / 2$, thus applying Theorem 2.11 we get that there exists an integer $z=p^{e} \geq 3$ such that each affine line with direction in $D_{\mathcal{U}}$ intersects $\mathcal{U} \cup \mathcal{P}$ in $0(\bmod z)$ points. We can apply Lemma 2.15 with $F=\ell \backslash$ and $E=\ell \backslash\left(\cup D_{\mathcal{U}}\right)$ to obtain $|E| \geq t$. Note 


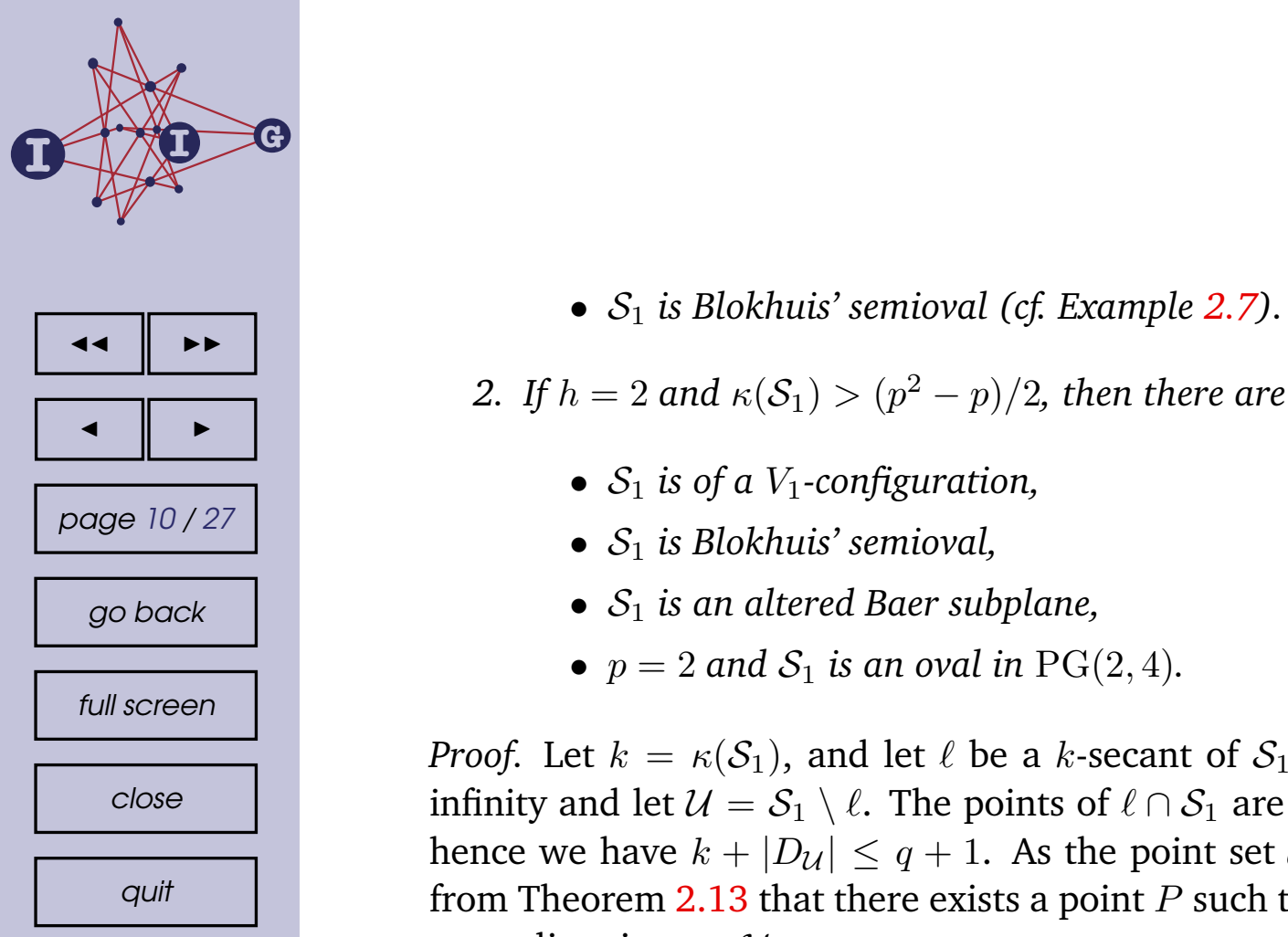

2. If $h=2$ and $\kappa\left(\mathcal{S}_{1}\right)>\left(p^{2}-p\right) / 2$, then there are four possibilities:

- $\mathcal{S}_{1}$ is of a $V_{1}$-configuration,

- $\mathcal{S}_{1}$ is Blokhuis' semioval,

- $\mathcal{S}_{1}$ is an altered Baer subplane,

- $p=2$ and $\mathcal{S}_{1}$ is an oval in $\mathrm{PG}(2,4)$.

Proof. Let $k=\kappa\left(\mathcal{S}_{1}\right)$, and let $\ell$ be a $k$-secant of $\mathcal{S}_{1}$. Consider $\ell$ as the line at infinity and let $\mathcal{U}=\mathcal{S}_{1} \backslash \ell$. The points of $\ell \cap \mathcal{S}_{1}$ are not determined directions, hence we have $k+\left|D_{\mathcal{U}}\right| \leq q+1$. As the point set $\mathcal{U}$ has size $q-1$, it follows from Theorem 2.13 that there exists a point $P$ such that $\mathcal{U} \cup\{P\}$ determines the same directions as $\mathcal{U}$.

First consider the case $h=1$. If $k>\min \{(p-3) / 2,(p+4) / 3\}$, then $\left|D_{\mathcal{U}}\right|<$ $\max \{\lfloor 2(p-1) / 3\rfloor+1,(p+5) / 2\}$ and thus Theorems 2.11 and 2.17 imply that either $\left|D_{\mathcal{U}}\right|=1$ and $\mathcal{U}$ is contained in a line, or $\left|D_{\mathcal{U}}\right|=(p+3) / 2$ and $\mathcal{U} \cup\{P\}$ is affinely equivalent to the graph of the function $x \mapsto x^{\frac{p+1}{2}}$. In the first case it is easy to see that $\mathcal{S}_{1}$ is a $V_{1}$-configuration. In the latter case the graph of $x \mapsto x^{\frac{p+1}{2}}$ is contained in two lines, namely $[1: 1: 0]$ and $[1:-1: 0]$, and these lines are $(p+1) / 2$-secants of $\mathcal{U} \cup\{P\}$. It is easy to see that $P$ has to be the point $(0: 0: 1)$, thus $\mathcal{S}_{1}$ has (at least) two $(p-1) / 2$-secants, and it is contained in a vertexless triangle. Such semiovals were characterized by Kiss and Ruff [28, Theorem 3.3]; it follows from their characterization that $\mathcal{S}_{1}$ must be Blokhuis' semioval.

Now suppose that $h=2$. If $k>\left(p^{2}-p\right) / 2$, then $\left|D_{\mathcal{U}}\right|<\left(p^{2}+p\right) / 2+1$, thus $\left|D_{\mathcal{U}}\right| \in\left\{1,\left(p^{2}+3\right) / 2\right\}$ or $1<\left|D_{\mathcal{U}}\right|<\left(p^{2}+3\right) / 2$. If $\left|D_{\mathcal{U}}\right|=1$ or $\left|D_{\mathcal{U}}\right|=$ $\left(p^{2}+3\right) / 2$, then we can argue as before. In the remaining case it follows from Theorems 2.11 and 1.3 (or already from [34, Theorem 5.7]), that $\left|D_{\mathcal{U}}\right|=p+1$ and $\mathcal{U} \cup\{P\} \cup D_{\mathcal{U}}$ is a Baer subplane. If $p>2$, then $\mathcal{S}_{1}$ has exactly $p^{2}-p-k$ tangents at each point of $\mathcal{U}$, hence $k=p^{2}-p-1$ and $\mathcal{S}_{1}$ is an altered Baer subplane. Finally, if $p=2$, then $k \geq 2$ and $\left|D_{\mathcal{U}}\right|=p+1=3$, thus $k=2$ and $\mathcal{S}_{1}$ is an oval in $\mathrm{PG}(2,4)$.

\section{Proof of the main lemma}

First we collect the most important properties of the Rédei polynomial. Consider a point set $\mathcal{U}=\left\{\left(a_{i}, b_{i}\right): i=1,2, \ldots,|\mathcal{U}|\right\}$ of the affine plane $\operatorname{AG}(2, q)$. The 
Rédei polynomial of $\mathcal{U}$ is

$$
H(X, Y)=\prod_{i=1}^{|\mathcal{U}|}\left(X+a_{i} Y-b_{i}\right) \in \mathrm{GF}(q)[X, Y]
$$

For any fixed value $y \in \mathrm{GF}(q)$, the univariate polynomial $H(X, y) \in \mathrm{GF}(q)[X]$ is fully reducible and it reflects some geometric properties of $\mathcal{U}$.

Lemma 3.1 (folklore). Let $H(X, Y)$ be the Rédei polynomial of the point set $\mathcal{U}$, and let $y \in \operatorname{GF}(q)$. Then $X=x$ is a root of $H(X, y)$ with multiplicity $r$ if and only if the line with equation $Y=y X+x$ meets $\mathcal{U}$ in exactly $r$ points.

We need another result on polynomials which will be crucial in the proof. For $r \in \mathbb{R}$, let $r^{+}=\max \{0, r\}$.

Theorem 3.2 (Szőnyi-Weiner Lemma, [37, Corollary 2.4], [23, Appendix, Result 6]). Let $f$ and $g$ be two-variable polynomials in $\operatorname{GF}(q)[X, Y]$. Let $d=\operatorname{deg} f$ and suppose that the coefficient of $X^{d}$ in $f$ is non-zero. For $y \in \operatorname{GF}(q)$, let $h_{y}=\operatorname{deg} \operatorname{gcd}(f(X, y), g(X, y))$, where gcd denotes the greatest common divisor of the two polynomials in $\mathrm{GF}(q)[X]$. Then for any $y_{0} \in \mathrm{GF}(q)$,

$$
\sum_{y \in \mathrm{GF}(q)}\left(h_{y}-h_{y_{0}}\right)^{+} \leq\left(\operatorname{deg} f(X, Y)-h_{y_{0}}\right)\left(\operatorname{deg} g(X, Y)-h_{y_{0}}\right) .
$$

A partial cover of $\mathrm{PG}(2, q)$ with $h>0$ holes is a set of lines in $\mathrm{PG}(2, q)$ such that the union of these lines covers all but $h$ points. We will also use the dual of the following result due to Blokhuis, Brouwer and Szőnyi [8].

Theorem 3.3 ([8, Proposition 1.5]). A partial cover of $\mathrm{PG}(2, q)$ with $h>0$ holes, not all on one line if $h>2$, has size at least $2 q-1-h / 2$.

Note that the following, main lemma is not restricted to $t$-semiarcs. The carrier of a pencil is the common point of the lines belonging to the pencil.

Lemma 3.4. Let $\mathcal{S}$ be a set of $s$ points in $\mathrm{PG}(2, q)$, let $\ell$ be a $k$-secant of $\mathcal{S}$ with $2 \leq k \leq q$, and let $1 \leq t \leq q-3$ be an integer. Suppose that through each point of $\mathcal{S} \cap \ell$ there pass exactly $t$ tangent lines to $\mathcal{S}$, and let $s=k+q-t+\varepsilon$ for some $\varepsilon \geq 0$. Let $A(n)$ be the set of those points in $\ell \backslash \mathcal{S}$ through which there pass at most $n$ skew lines to $\mathcal{S}$. Then the following hold.

- If $t=1$, then

1. $\varepsilon<\frac{k}{2}-1$ implies that the $k$ tangent lines at the points of $\mathcal{S} \cap \ell$ and the skew lines through the points of $A(2)$ belong to a pencil (hence $A(2) \backslash A(1)$ is empty), 




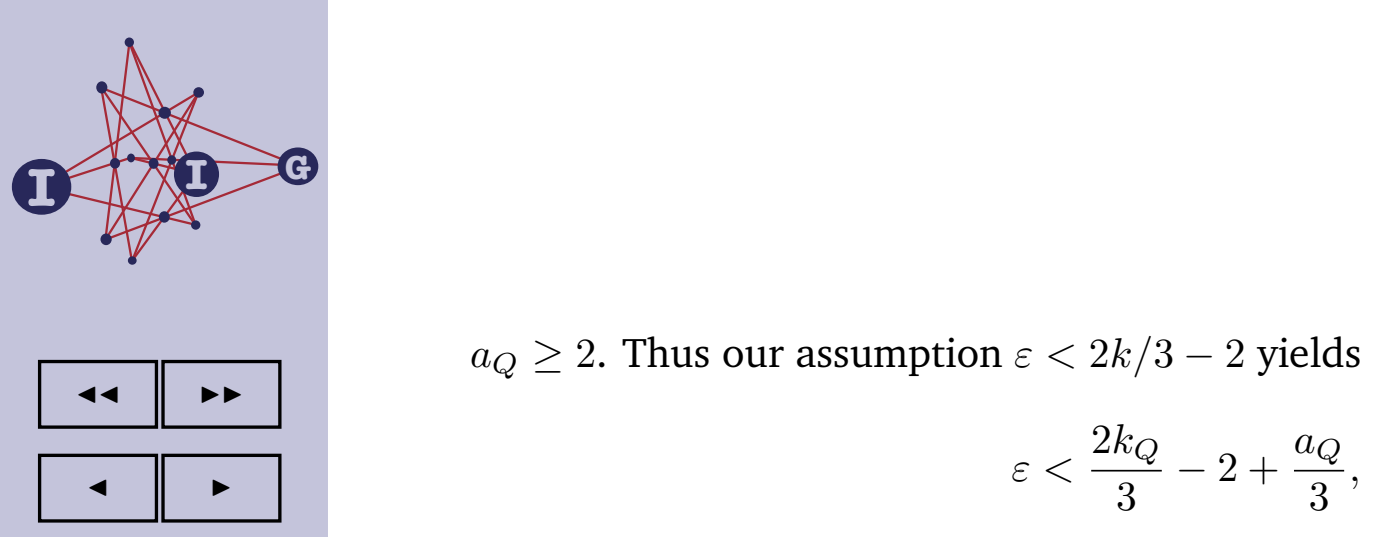

and so (2) gives a contradiction for $3 \leq \operatorname{ind}_{Q}(P) \leq k_{Q}+a_{Q}-2-\varepsilon$.

In cases (a) and (c) it follows that the lines of $\mathcal{L}_{Q}$ either form a dual arc and we are finished, or there is a point $R$ with $Q$-index at least $k_{Q}+a_{Q}-1-\varepsilon$. In case (b) either $\mathcal{L}$ is a dual arc and we are finished, or there is a point $R$ with index at least $q-1-\varepsilon$ (since in this case $k_{Q}+a_{Q}=q$ ). So it remains to handle the case when such a point $R$ exists. Let $\mathcal{B}=(\ell \backslash(\mathcal{S} \cup A(2))) \cup(\mathcal{S} \backslash \ell) \cup R$ and denote by $h$ the number of lines of $\operatorname{PG}(2, q)$ not blocked by $\mathcal{B}$. It is easy to see that, apart from $\ell, \mathcal{B}$ blocks all but at most $(k+2|A(2)|)-\left(k_{Q}+a_{Q}-1-\varepsilon\right)$ lines of $\operatorname{PG}(2, q)$. In case (a) $\mathcal{B}$ blocks $\ell$ and $k+|A(2)|=k_{Q}+a_{Q}$, hence

$$
h \leq|A(2)|+1+\varepsilon
$$

In cases (b) and (c) $\mathcal{B}$ does not block $\ell$ and $k+|A(2)|=\left(k_{Q}+a_{Q}\right)+1$, thus

$$
h \leq|A(2)|+3+\varepsilon
$$

Suppose to the contrary that these $h$ lines do not pass through one point. Then by the dual of Theorem 3.3 we have

$$
|\mathcal{B}|=q+1-(k+|A(2)|)+(q-1+\varepsilon)+1 \geq 2 q-1-h / 2 .
$$

Rearranging it we obtain $\varepsilon \geq k+|A(2)|-2-h / 2$. In case (a), together with (6), this would imply $\varepsilon \geq 2 k / 3-5 / 3+|A(2)| / 3$. In cases (b) and (c), together with (7), $\varepsilon \geq(q+k) / 3-2$ would follow. Both cases yield a contradiction because of our assumption on $\varepsilon$. Hence the corresponding lines can be blocked by $R$ and one more point, thus they belong to two pencils.

Although the forthcoming applications in this paper all use Lemma 3.4, we shall give another, more general but less detailed result whose proof is based on the very same ideas.

Theorem 3.5. Suppose that $\mathcal{S} \subset \mathrm{PG}(2, q)$ is a point set, $\ell$ is a line, and let $s=|\mathcal{S} \backslash \ell|$. Let $\mathcal{K} \subset \ell \backslash \mathcal{S}$ be a set of $k \leq q$ points. Denote by $\mathcal{L}$ the set of skew lines to $\mathcal{S}$ through the points of $\mathcal{K}$, not including $\ell$, and let $\delta=|\mathcal{L}|$. Let $m$ be an integer such that any point of $\mathcal{K}$ is incident with at most $m$ lines of $\mathcal{L}$. Suppose that there exists an integer $t$ such that $(t-1) q+m<\delta<(t+1)(k+q-s-t-1)$. If $\delta<(n+1)(k+q-s-t-n / 2)$ for some integer $n$, then the lines of $\mathcal{L}$ belong to $n$ pencils whose carriers are not on $\ell$. 
Proof. Let the index of a point $P$, ind $(P)$, be the number of lines of $\mathcal{L}$ incident with $P$. Similarly as in the proof of Lemma 3.4, let $P \in \operatorname{PG}(2, q) \backslash(\mathcal{S} \cup \ell)$; we may assume that $\ell$ is the line $[1: 0: 0], P=\left(y_{0}\right) \in \ell_{\infty} \backslash\left\{Y_{\infty}\right\}$ and $Y_{\infty} \notin \mathcal{K}$. Let $D=\left(\ell_{\infty} \backslash\left\{Y_{\infty}\right\}\right) \cap \mathcal{S},|D|=d$ and let $\mathcal{U}=\mathcal{S} \backslash\left(\ell \cup \ell_{\infty}\right)$. Again, let $g(X, Y)$ and $f(X, Y)$ be the Rédei polynomials of $\mathcal{K}$ and $\mathcal{U}$; their degrees are $k$ and $s-d$, respectively. Let $\bar{D}=\ell_{\infty} \backslash\left(D \cup\left\{Y_{\infty}\right\}\right)$. Then for any point $(y) \in \bar{D}$,

$$
h_{y}:=\operatorname{deg} \operatorname{gcd}(f(X, y), g(X, y))=k-\operatorname{ind}(y) .
$$

Applying the Szőnyi-Weiner Lemma we get

$$
(q-d) \operatorname{ind}\left(y_{0}\right)-\delta=\sum_{(y) \in \bar{D}}\left(\operatorname{ind}\left(y_{0}\right)-\operatorname{ind}(y)\right) \leq \operatorname{ind}\left(y_{0}\right)\left(s-d-k+\operatorname{ind}\left(y_{0}\right)\right) .
$$

After rearranging it we obtain

$$
0 \leq \operatorname{ind}(P)^{2}-\operatorname{ind}(P)(q+k-s)+\delta .
$$

Assuming ind $(P)=t+1$, (8) contradicts $\delta<(t+1)(k+q-s-t-1)$, hence either $\operatorname{ind}(P) \leq t$ or $\operatorname{ind}(P) \geq q+k-s-t$. Suppose that there is a line of $\mathcal{L}$ containing no point with large index. Then $\delta \leq m+q(t-1)$ follows, a contradiction. Hence the lines of $\mathcal{L}$ are blocked by the points with large index. If there were at least $n+1$ such points, then $\delta \geq(n+1)(q+k-s-t)-\left(\begin{array}{c}n+1 \\ 2\end{array}\right)$ would follow, contradicting $\delta<(n+1)(k+q-s-t-n / 2)$.

In [36, Section 3], among other techniques, Szőnyi and Weiner also use their lemma (Lemma 3.2) in basically the same way to derive a result roughly saying that if a small point set has only a few skew lines to it, then it can be extended to a blocking set by adding a few points to it. Now, with the notation of Theorem 3.5, extending the set $(\mathcal{S} \cup \ell) \backslash \mathcal{K}$ to a blocking set by adding $n$ points to it is equivalent to finding $n$ pencils that contain the lines of $\mathcal{L}$. However, the points found using the result of [36] might also be on $\ell$. Now let us give some immediate consequences of Lemma 3.4.

Corollary 3.6. Let $\mathcal{S}_{1}$ be a semioval in $\mathrm{PG}(2, q)$ and let $\ell$ be a $k$-secant of $\mathcal{S}_{1}$. If $\left|\mathcal{S}_{1}\right|<q+\frac{3 k}{2}-2$, then the $k$ tangent lines at the points of $\mathcal{S}_{1} \cap \ell$ belong to a pencil. If $\left|\mathcal{S}_{1}\right|<q+\frac{5 k}{3}-3$, then the $k$ tangent lines at the points of $\mathcal{S}_{1} \cap \ell$ either belong to two pencils or they form a dual $k$-arc.

If $k=q-1$, then we get a stronger result than the previous characterization of Kiss [26, Corollary 3.1].

Corollary 3.7. Let $\mathcal{S}_{1}$ be a semioval in $\mathrm{PG}(2, q)$. If $\mathcal{S}_{1}$ has a $(q-1)$-secant $\ell$ and $\left|\mathcal{S}_{1}\right|<\frac{5 q}{2}-\frac{7}{2}$ holds, then $\mathcal{S}_{1}$ is contained in a vertexless triangle and it has two $(q-1)$-secants. 


$\left|\ell^{\prime} \cap(\mathcal{P} \cup \mathcal{U})\right| \equiv 0(\bmod z)$. Hence the two required properties of Lemma 2.15 hold, thus $|A(t)| \geq t$.

To proceed, we need some results on semiarcs with two long secants proved by Csajbók.

Lemma 4.4 ([15, Theorem 13]). Let be a t-semiarc in the projective plane $\Pi_{q}$, go back

full screen

close

quit $1<t<q$. Suppose that there exist two lines $\ell_{1}$ and $\ell_{2}$ such that $\left|\ell_{1} \backslash\left(\cup \ell_{2}\right)\right|=n$ and $\left|\ell_{2} \backslash\left(\cup \ell_{1}\right)\right|=m$. If $\ell_{1} \cap \ell_{2} \notin$, then $n=m=t$ or $q \leq \min \{n, m\}+2 n m /(t-1)$.

We cite only three particular cases of the complete characterization of $t$-semiarcs in $\mathrm{PG}(2, q)$ with two $(q-t)$-secants whose common point is not in the semiarc. Such semiarcs are called semiarcs of $V_{t}^{\circ}$ type. Note that the tight semiarcs of $V_{t}^{\circ}$ type are precisely the $V_{t}$-configurations.

Theorem 4.5 ([15, Theorem 22]). Let be a t-semiarc of $V_{t}^{\circ}$ type in $\mathrm{PG}(2, q)$, $q=p^{h}$, p prime, and let $t \leq q-2$. Then the following hold.

1. If $\operatorname{gcd}(q, t)=1$, then is contained in a vertexless triangle.

2. If $\operatorname{gcd}(q, t)=1$ and $\operatorname{gcd}(q-1, t-1)=1$, then is a $V_{t}$-configuration.

3. If $\operatorname{gcd}(q-1, t)=1$, then is contained either in a vertexless triangle, or in the union of three concurrent lines with their common point removed.

Now we are ready to prove our main characterization theorems for small semiarcs with a long secant. We distinguish two cases as the results on blocking sets in $\mathrm{PG}(2, q)$ are stronger if $q$ is a prime.

Theorem 4.6. Let be a $t$-semiarc in $\mathrm{PG}(2, p)$, p prime.

1. If $t=1, p \geq 5$ and $\kappa\left(\mathcal{S}_{1}\right) \geq \frac{p-1}{2}$, then

- $\mathcal{S}_{1}$ is contained in a vertexless triangle and has two $(p-1)$-secants, or

- $\mathcal{S}_{1}$ is projectively equivalent to Blokhuis' semioval, or

- $\left|\mathcal{S}_{1}\right| \geq \min \left\{\frac{3 \kappa\left(\mathcal{S}_{1}\right)}{2}+p-2,2 \kappa\left(\mathcal{S}_{1}\right)+\frac{p+1}{2}\right\}$.

2. If $t=2, p \geq 7$ and $\kappa\left(\mathcal{S}_{2}\right) \geq \frac{p+3}{2}$, then

- $\mathcal{S}_{2}$ is a $V_{2}$-configuration, or

- $\left|\mathcal{S}_{2}\right| \geq \min \left\{\frac{4 \kappa\left(\mathcal{S}_{2}\right)}{3}+p-3,2 \kappa\left(\mathcal{S}_{2}\right)+\frac{p-1}{2}\right\}$. 
Then Theorem 1.3 implies that it contains a line $\ell_{1}$. Note that $\ell_{1} \cap \ell \notin$. Since cannot have more than $p-t$ collinear points, by the construction of $\mathcal{B}_{t+1}(, \ell)$ we have that $\ell_{1}$ is a $(p-t)$-secant or a $(p-t-1)$-secant of , and hence so is $\ell$. Then (using $p \geq 23$ and $t<\sqrt{p}$ ) Lemma 4.4 implies that both $\ell$ and $\ell_{1}$ are $(p-t)$-secants. Since $\operatorname{gcd}(t, p)=1$, Theorem 4.5 implies that is contained in a vertexless triangle.

For non-prime values of $q$, our next theorem roughly says that if $t$ is small, then small $t$-semiarcs with a long secant are of $V_{t}^{\circ}$ type. If $q$ is a square, then we can characterize altered Baer subplanes (Example 2.10) as well. Recall that altered Baer subplanes are $t$-semiarcs of size $(q-\sqrt{q}-t)+(q-t)$ with a $(q-$ $\sqrt{q}-t)$-secant.

Theorem 4.7. Let be a t-semiarc in $\mathrm{PG}(2, q), q=p^{h}, h \geq 2$ if $p$ is an odd prime and $h \geq 6$ if $p=2$. Suppose that

$$
\kappa() \geq \begin{cases}q-\sqrt{q}-t & \text { if } h \text { is even, } \\ q-c_{p} q^{2 / 3}-t & \text { if } h \text { is odd, }\end{cases}
$$

where $c_{p}=2^{-1 / 3}$ for $p=2,3$ and $c_{p}=1$ for $p>3$ (cf. Theorem 1.3). Then the following hold.

1. If $h=2 d$ and $t<(\sqrt{5}-1)(\sqrt{q}-1) / 2$, then

- $\|<2 \kappa()+\sqrt{q}$ implies that is of $V_{t}^{\circ}$ type;

- $\|=2 \kappa()+\sqrt{q}$ and $q>9$ implies that is either of $V_{t}^{\circ}$ type or an altered Baer subplane.

2. If $h=2 d+1, \|<2 \kappa()+c_{p} q^{2 / 3}$ and $t<q^{1 / 3}-3 / 2$ (or $t<(2 q)^{1 / 3}-2$ when $p=2,3)$, then is of $V_{t}^{\circ}$ type.

Proof. Let $k=\kappa()$ and let $\ell$ be a $k$-secant of . Note that as $t$ is small enough, Theorem 2.3 implies that $k \leq q-t$. We define $A(n) \subset \ell$ as usual. To apply Lemma 3.4, we need $k>q-\frac{q}{t}+1$; furthermore, $\varepsilon<k / 2-1$ for $t=1$ and $\varepsilon<k /(t+1)-t / 2$ for $t \geq 2$. Let us first consider the condition on $k$. If $q$ is a square, then $k \geq q-\sqrt{q}-t>q-\frac{q}{t}+1$ holds if $t<\Phi(\sqrt{q}-1)$, where $\Phi=\frac{\sqrt{5}-1}{2} \approx 0.618034$. If $q$ is not a square, then $t<q^{1 / 3}-3 / 2$ (or $t<(2 q)^{1 / 3}-2$ when $p=2,3$ ) and $k \geq q-c_{p} q^{2 / 3}-t$ imply $k>q-\frac{q}{t}+1$.

Next we treat the condition on $\varepsilon$. Let us define $b(q)$ as follows:

$$
b(q):= \begin{cases}\sqrt{q} & \text { if } h \text { is even } \\ c_{p} q^{2 / 3} & \text { if } h \text { is odd. }\end{cases}
$$


As $\|=k+q-t+\varepsilon,\| \leq 2 k+b(q)$ implies $\varepsilon \leq k-q+b(q)+t$.

Suppose first that $t \geq 2$. As $\varepsilon \leq k-q+b(q)+t$, it is enough to prove $k-q+b(q)+t<\frac{k}{t+1}-\frac{t}{2}$. After rearranging we get that this is equivalent to

$$
k<(q-t)+\left(\frac{q-b(q)}{t}-\frac{t}{2}-b(q)-\frac{3}{2}\right),
$$

thus it is enough to see (as $k \leq q-t$ holds automatically) that

$$
\frac{q-b(q)}{t}-\frac{t}{2}-b(q)-\frac{3}{2}>0
$$

As a function of $t$ the left hand side decreases monotonically. It is positive when $t$ is maximal (under the respective assumptions), hence the condition of Lemma 3.4 on $\varepsilon$ is satisfied for $t \geq 2$.

If $t=1$, then the upper bounds on $t$ imply $q \geq 9$ for $h=2 d$ and $q \geq 27$ for $h=2 d+1$. From these lower bounds on $q$ and from $k \leq q-1$ it follows that $k / 2 \leq(q-1) / 2 \leq q-b(q)-2$, whence we obtain $k-q+b(q)+1 \leq \frac{k}{2}-1$. If $\left|\mathcal{S}_{1}\right|<2 k+b(q)$, then $\varepsilon<k-q+b(q)+1 \leq \frac{k}{2}-1$. If $\left|\mathcal{S}_{1}\right|=2 k+b(q)$, then $\varepsilon=k-q+b(q)+1$ and we are in the case $h=2 d$; here the assumption $q>9$ implies $\varepsilon=k-q+b(q)+1<\frac{k}{2}-1$. Thus the condition of Lemma 3.4 on $\varepsilon$ also holds for $t=1$.

For $\|<2 k+b(q)$, we prove the $h$ even and $h$ odd cases of the theorem simultaneously. Construct the blocking set $\mathcal{B}_{t}(, \ell)$ as in Lemma 4.1. The conditions in Lemma 4.3 hold, hence the size of $A(t)$ is at least $t$. The size of $\mathcal{B}_{t}(, \ell)$ is $2 q+1+\varepsilon-k-|A(t)|<q+b(q)+1$, thus Theorem 1.3 implies that $\mathcal{B}_{t}(, \ell)$ contains a line $\ell_{1}$. Since cannot have more than $q-t$ collinear points, by the construction of $\mathcal{B}_{t}(, \ell)$ we get that $\ell_{1}$ is a $(q-t)$-secant of , and hence so is $\ell$. Thus is of $V_{t}^{\circ}$ type.

Now consider the case $\|=2 k+\sqrt{q}$ (hence $\varepsilon=k-q+\sqrt{q}+t$ ), and suppose that does not have two $(q-t)$-secants. We can repeat the above arguing and get that $\mathcal{B}_{t}(, \ell)$ is a Baer subplane because of Theorem 1.3. Then $\left|\mathcal{B}_{t}(, \ell)\right|=$ $q+\sqrt{q}+1=2 q+1+\varepsilon-k-|A(t)|$ yields $|A(t)|=t$. The size of $\ell \cap \mathcal{B}_{t}(, \ell)$ is either 1 or $\sqrt{q}+1$. In the latter case $k=q-\sqrt{q}-t$ and is an altered Baer subplane. In the first case $k=q-t$; we show that this cannot occur. Denote by $R$ the common point of $\ell$ and $\mathcal{B}_{t}(, \ell)$ and let $P$ be any point of $\mathcal{B}_{t}(, \ell) \backslash(\ell \cup)$. Among the lines of the Baer subplane $\mathcal{B}_{t}(, \ell)$ there are $\sqrt{q}+1$ lines incident with $P$. One of them is $P R$, which meets in at least $\sqrt{q}-t>1$ points; each of the other $\sqrt{q}$ lines of the subplane intersects in at least $\sqrt{q}+1-t>1$ points. Thus 


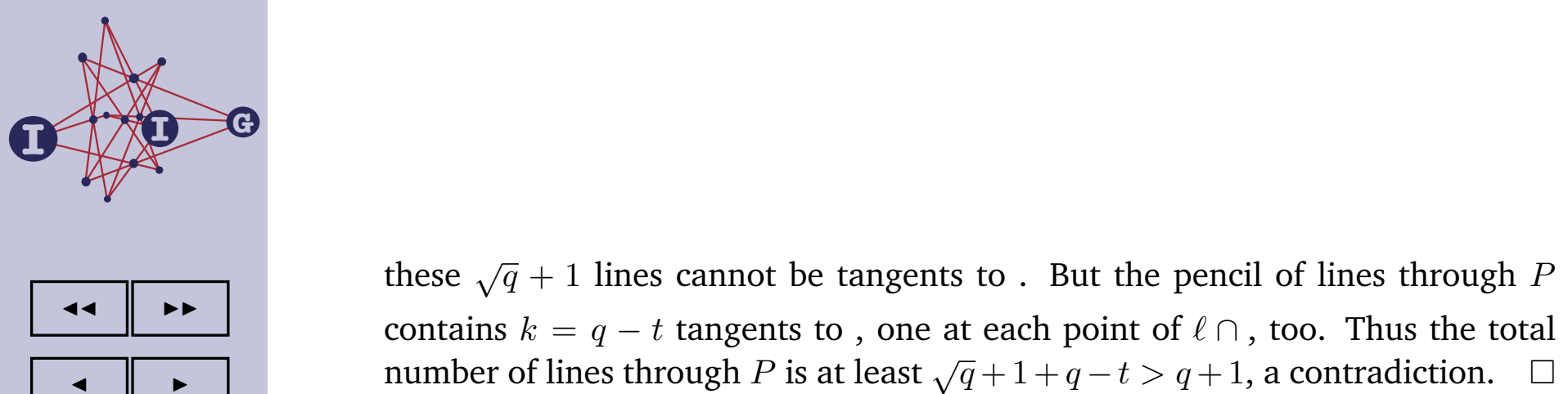
number of lines through $P$ is at least $\sqrt{q}+1+q-t>q+1$, a contradiction.

\section{References}

[1] S. Ball, The number of directions determined by a function over a finite field, J. Combin. Theory Ser. A 104 (2003), 341-350.

[2] D. Bartoli, On the structure of semiovals of small size, J. Combin. Des. 22 (2014), 525-536.

[3] L. M. Batten, Determining sets, Australas. J. Combin. 22 (2000), 167-176.

[4] A. Blokhuis, On the size of a blocking set in $\mathrm{PG}(2, p)$, Combinatorica 14 (1994), 111-114.

[5] Characterization of seminuclear sets in a finite projective plane, J. Geom. 40 (1991), 15-19.

[6] A. Blokhuis, S. Ball, A. E. Brouwer, L. Storme and T. Szőnyi, On the number of slopes of the graph of a function defined on a finite field, J. Combin. Theory Ser. A 86 (1999), 187-196.

[7] A. Blokhuis, A. E. Brouwer and T. Szőnyi, The number of directions determined by a function $f$ on a finite field, J. Combin. Theory Ser. A 70 (1995), 349-353.

[8] , Covering all points except one, J. Algebraic Combin. 32 (2010), 59-66.

[9] A. Blokhuis, Á. Seress and H. A. Wilbrink, On sets of points in PG $(2, q)$ without tangents. Proceedings of the First International Conference on Blocking Sets (Giessen, 1989). Mitt. Math. Sem. Giessen 201 (1991), 3944.

[10] A. Blokhuis, L. Storme and T. Szőnyi, Lacunary polynomials, multiple blocking sets and Baer subplanes, J. London Math. Soc. 60 (1999), 321332.

[11] A. Blokhuis, T. Szőnyi and Zs. Weiner, On sets without tangents in Galois planes of even order. Proceedings of the Conference on Finite Geometries (Oberwolfach, 2001). Des. Codes Cryptogr. 29 (2003), no. 1-3, 91-98. 
[12] A. E. Brouwer and A. Schrijver, The blocking number of an affine space, J. Combin. Theory Ser. A 24 (1978), 251-253.

[13] A. A. Bruen, Baer subplanes and blocking sets, Bull. Amer. Math. Soc. 76 (1970), 342-344.

[14] R. Calderbank and W. M. Kantor, The geometry of two-weight codes, Bull. London Math. Soc. 18 (1986), no. 2, 97-122.

[15] B. Csajbók, Semiarcs with long secants, Electron. J. Combin. 21 (2014), \# P1.60, 14 pages.

[16] B. Csajbók and Gy. Kiss, Notes on semiarcs, Mediterr. J. Math. 9 (2012), 677-692.

[17] M. De Boeck and G. Van de Voorde, A linear set view on KM-arcs, submitted (2014).

[18] J. M. Dover, Semiovals with large collinear subsets, J. Geom. 69 (2000), 58-67.

[19] A. Gács, On a generalization of Rédei's theorem, Combinatorica 23 (2003), 585-598.

[20] A. Gács, L. Lovász and T. Szőnyi, Directions in $\mathrm{AG}\left(2, p^{2}\right)$, Innov. Incidence Geom. 6/7 (2009), 189-201.

[21] A. Gács and Zs. Weiner, On $(q+t, t)$-arcs of type $(0,2, t)$, Des. Codes Cryptogr. 29 (2003), 131-139.

[22] M. Giulietti and E. Montanucci, On hyperfocused arcs in PG(2,q), Discrete Math. 306 (2006), no. 24, 3307-3314.

[23] T. Héger, Some graph theoretic aspects of finite geometries, $\mathrm{PhD}$ Thesis, Eötvös Loránd University (2013).

[24] J. W. P. Hirschfeld, Projective Geometries over Finite Fields, $2^{\text {nd }}$ ed., Clarendon Press, Oxford, 1998.

[25] R. E. Jamison, Covering finite fields with cosets of subspaces, J. Combin. Theory Ser. A 22 (1977), 253-266.

[26] Gy. Kiss, Small semiovals in PG(2, q), J. Geom. 88 (2008), 110-115. , A survey on semiovals, Contrib. Discrete Math. 3 (2008), 81-95.

[28] Gy. Kiss and J. Ruff, Notes on small semiovals, Ann. Univ. Sci. Budapest. Eötvös Sect. Math. 47 (2004), 97-105. 
[29] G. Korchmáros and F. Mazzocca, On $(q+t, t)$-arcs of type $(0,2, t)$ in a Desarguesian plane of order q, Math. Proc. Cambridge Philos. Soc. 108 (1990), 445-459.

[30] L. Lovász and A. Schrijver, Remarks on a theorem of Rédei, Studia Scient. Math. Hungar. 16 (1981), 449-454.

[31] O. Polverino, Small minimal blocking sets and complete $k$-arcs in PG $\left(2, p^{3}\right)$, Discrete Math. 208/209 (1999), 469-476.

[32] P. Sziklai, On small blocking sets and their linearity, J. Combin. Theory Ser. A 115 (2008), 1167-1182.

[33] , Subsets of $\mathrm{GF}\left(q^{2}\right)$ with $d$-th power differences, Discrete Math. 208/209 (1999), 547-555.

[34] T. Szőnyi, Blocking Sets in Desarguesian Affine and Projective Planes, Finite Fields Appl. 3 (1997), 187-202.

[35] _ On the number of directions determined by a set of points in an affine Galois plane, J. Combin. Theory Ser. A 74 (1996), 141-146.

[36] T. Szőnyi and Zs. Weiner, On the stability of small blocking sets, J. Algebraic Combin. 40 (2014), 279-292.

[37] _ Proof of a conjecture of Metsch, J. Combin. Theory Ser. A 118 (2011), 2066-2070.

[38] _ On the stability of sets of even type, Adv. Math. 267 (2014), 381394.

[39] P. Vandendriessche, Codes of Desarguesian projective planes of even order, projective triads and $(q+t, t)$-arcs of type $(0,2, t)$, Finite Fields Appl. 17 (2011), 521-531.

Bence Csajbók

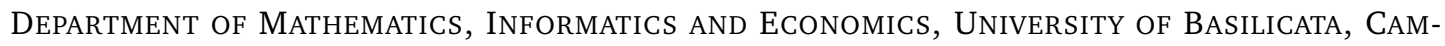
pus Macchia Romana, Via Dell'Ateneo LuCANo, I-85100 Potenza, Italy

AND

MTA-ELTE GEOMEtric ANd Algebraic COMbinatorics ResEArch Group, EÖtvÖs LorÁnd UniVERSITY, 1117 BudAPEST, PÁZMÁNy PÉTER SÉTÁNy 1/C, HuNGARY

e-mail: csajbokb@cs.elte.hu

website: http://www.cs.elte.hu/ csajbokb

Tamás Héger 

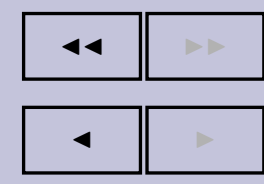

page $27 / 27$

go back

full screen

close

quit
DEPARTMENT OF COMPUTER SCIENCE, AND

MTA-ELTE Geometric And Algebraic Combinatorics Research Group, EÖtvös LoRÁnd UniVERSITY, 1117 BUdAPEST, PÁZMÁNY PÉTER SÉTÁNY 1/C, HuNGARY

e-mail: heger@cs.elte.hu

website: http://www.cs.elte.hu/ hetamas

György Kiss

DEPARTMENT OF GEOMETRY, AND

MTA-ELTE GeOMETRIC AND Algebraic Combinatorics Research Group, EÖtvös LorÁND UniVERSITY, 1117 BUdAPEST, PÁZMÁNY PÉTER SÉTÁNY 1/C, HUNGARY

e-mail: kissgy@cs.elte.hu 